\title{
Publisher Correction: Non-invasive assessment of hepatic mitochondrial metabolism by positional isotopomer NMR tracer analysis (PINTA)
}

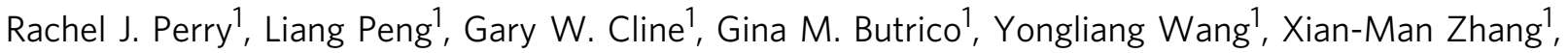
Douglas L. Rothman ${ }^{2,3}$, Kitt Falk Petersen ${ }^{1} \&$ Gerald I. Shulman (1) 1,4,5

Correction to: Nature Communications https://doi.org/10.1038/s41467-017-01143-w, published online: 06 Oct 2017

The originally published version of this Article contained an error in Equation 30, which was inadvertently introduced during the production process. The incorrect form of Equation 30 was:

$$
\frac{V_{P C}}{V_{C S}}=\left(\left[\frac{\left[3-{ }^{13} C\right] O A A}{\left[1-{ }^{13} C\right] O A A}-1\right] * \frac{1}{1-0.5 * K_{3}} * \frac{1}{2}\right)
$$

The correct form of Equation 30 is:

$$
\frac{V_{P C}}{V_{C S}}=\left[0.5 * \frac{\left[3-{ }^{13} C\right] O A A}{\left[1-{ }^{13} C\right] O A A}-1\right] * \frac{1}{1-0.5 * K_{3}}
$$

This has now been corrected in the PDF and HTML versions of the Article.

Published online: 31 January 2018

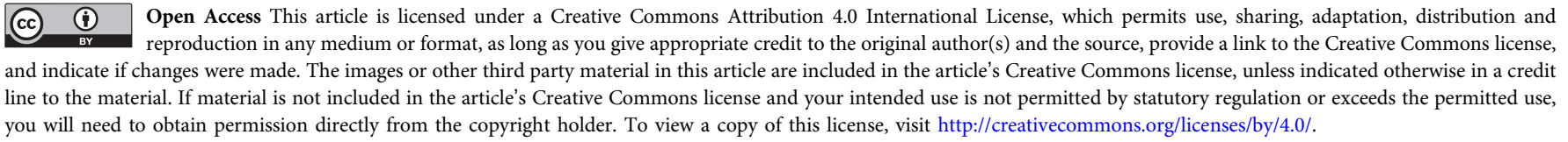

(C) The Author(s) 2018

\footnotetext{
${ }^{1}$ Departments of Internal Medicine, Yale University School of Medicine, New Haven, CT 06520, USA. ${ }^{2}$ Departments of Radiology and Biomedical Imaging, Yale University School of Medicine, New Haven, CT 06520, USA. ${ }^{3}$ Departments of Biomedical Engineering, Yale University School of Medicine, New Haven, CT 06520, USA. ${ }^{4}$ Departments of Cellular and Molecular Physiology, Yale University School of Medicine, New Haven, CT 06520, USA. ${ }^{5}$ Departments of Howard Hughes Medical Institute, Yale University School of Medicine, New Haven, CT 06520, USA. Correspondence and requests for materials should be addressed to G.I.S. (email: gerald.shulman@yale.edu)
} 